\title{
Triple drug combination of telmisartan, amlodipine and hydrochlorothiazide in the treatment of essential hypertension
}

\author{
Manish Maladkar', Vijay Kumar Verma ${ }^{2}$, Keshav A. Narsikar ${ }^{3}$, Rajan D. Walinjkar ${ }^{4}$, W. R. Patil ${ }^{5}$, \\ N. J. S. Saggu ${ }^{6}$, Suresh P. Kulkarni ${ }^{7}$

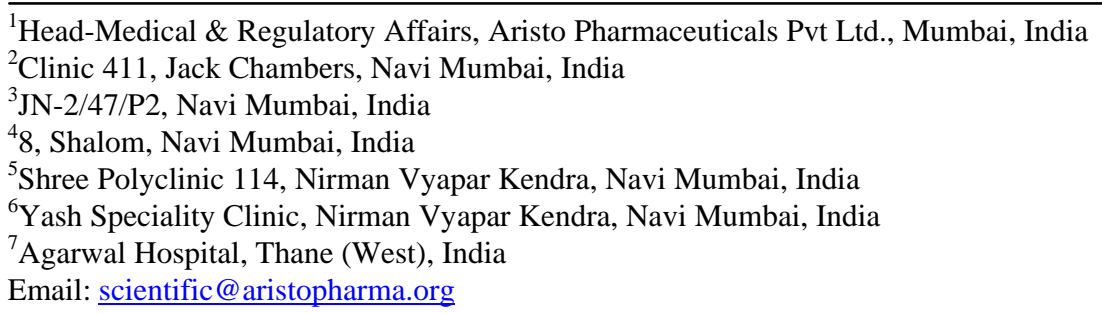

Received 26 December 2011; revised 28 February 2012; accepted 9 March 2012

\section{ABSTRACT}

Background: Triple drug therapy comprising angiotensin receptor blocker (ARB), calcium channel blocker (CCB) and hydrochlorothiazide (HCT) effectively controls essential hypertension as evident from the literature. This study was undertaken to assess the efficacy and safety of triple combination compared to the dual drug therapy. Methodologies: A total of 220 male and female patients with essential hypertension were enrolled in the study. The patients were divided into two groups. Group A received a bilayer tablet of FDC of Telmisartan + Amlodipine + HCT and group B received FDC tablet of Telmisartan + HCT. Both the treatments were administered once daily for twelve weeks. The patients were asked to follow-up on week 1, 2, 4, 6, 8 and 10 for periodic efficacy and safety evaluations. Effect on systolic blood pressure (SBP), diastolic blood pressure (DBP) and quality of life (QOL) were recorded during the course of the trial. Results: Blood pressure reduction (BP) to the desired goals was observed with both the treatments. The SBP and DBP reductions were superior in triple combination therapy than double combination. Both treatments improved QOL of patients. Conclusion: Triple drug combination of telmisartan, amlodipine and HCT may serve a potential role in achieving desired BP goals, in patients with essential hypertension, which are otherwise poorly managed by either monotherapy or dual drug therapy.

Keywords: Essential Hypertension; Telmisartan;
Amlodipine and HCT

\section{INTRODUCTION}

Treatment of essential hypertension has largely evolved from single drug therapy to combinations of drugs exhibiting different mechanisms of action. The prime objective for such experiments is to reduce BP effectively, as it reduces mortality and morbidity associated with stroke and coronary heart disease. Especially in patients with concomitant risk factors like diabetes, optimum values for BP is the need of the hour. In spite of many therapy advancements, effective BP control is far away from reality in many patients. A report says that only $37 \%$ of hypertensive patients in the US achieve the conservative goal of $<140 / 90 \mathrm{mmHg}$ of BP [1]. Multi drug combinations are the possible answers for the treatment of such difficult to control BP patients [2]. Many combinations have been tried in clinical trials and few are available worldwide. ARBs like losartan, olmesartan and telmisartan, were evaluated in combination with either HCT or CCB for their use in essential hypertension. Most of the clinical evidences showed superior efficacy of such combinations over monotherapies, with modest adverse events being reported [3,4] ARB-HCT combinations are getting increasingly popular in the antihypertensive therapy and prescriptions are continuing to be switched to these combinations. In recent times, more than two drugs were also combined for better clinical outcomes. ARBCCB-HCT fixed dose combinations were also developed on the same lines. Olmesartan-amlodipine-HCT has been studied in randomized clinical trials for its efficacy \& 
safety and it has been concluded that this triple combination is superior in BP reduction, compared to dual combinations and it also showed comparable tolerability. $[5,6]$.

The present study was undertaken to determine the BP lowering effect of telmisartan when combined with amlodipine and HCT compared to dual combination of telmisartan and amlodipine.

Telmisartan exerts its BP lowering effect by blocking angiotensin II receptors. In addition, telmisartan also has PPAR-gamma (Peroxisome Proliferator Activator Receptor) agonistic activity; this could be a potential reason for the better clinical outcomes with this molecule compared to other ARBs. Apart from effective BP lowering, telmisartan is also associated with renal protection, improved ventricular remodelling, reduced inflammation and oxidative stress, etc. [7-10]. Many authors have demonstrated the superiority of telmisartan when combined with other antihypertensives like amlodipine or HCT compared to monotherapy. [11-14]. Telmisartan combinations were also better in BP reduction compared to other ARB combinations [15]. Amlodipine is one of the most commonly used CCBs in the management of patients with hypertension. Amlodipine also exhibits plaque stabilisation properties and may prevent more number of events of stroke and myocardial infarction (MI) as compared to ARB [16,17]. HCT is a thiazide diuretic which helps to reduce BP by volume depletion. It has a long history of usage and has been tried in combination with many other antihypertensive drugs.

\section{MATERIALS AND METHODS}

This randomized, single blind study was conducted in accordance with ICH guidelines and declaration of Helsinki. A total of 220 male and female patients with essential hypertension were enrolled in the study, as per the inclusion/exclusion criteria defined in the protocol. All the patients were screened for their laboratory parameters, vital organ functions and BP (both sitting and supine) prior to enrolment. Patients with SBP $140-200 \mathrm{mmHg}$ and DBP 90 - $120 \mathrm{mmHg}$ were considered for the study. The patients were divided into two groups. Group A received a bilayer tablet of FDC of Telmisartan (40 mg) + Amlodipine (5 mg) + HCT (12.5 mg) and group B received FDC tablet of Telmisartan (40 mg) + HCT (12.5 $\mathrm{mg})$. Both the treatments were administered once daily for twelve weeks. The patients were asked to follow-up on week 1, 2, 4, 6, 8 and 10 for periodic efficacy and safety evaluations. Effect on both SBP and DBP was recorded during the course of the trial. Both the therapies were also evaluated on the basis of Quality of Life (QOL) questioner and safety parameters. QOL was assessed by using a questionnaire based on the common problems associated with elevated BP. The p values for evaluation parameters were calculated using single factor ANOVA.

\section{RESULTS}

The baseline parameters for both the groups were not statistically different. Total number of 208 patients completed the study with 12 patients considered as dropouts as they lost to follow up. A total number of 106 patients from group A and 102 patients of group B were taken for final analysis. The vital signs like body temperature, pulse rate and respiratory rate remained unchanged throughout the follow up visits in both the groups.

\subsection{The Effect of Triple Drug Combination on Systolic Blood Pressure (SBP)}

SBP measured at sitting and supine postures dropped considerably with both the treatments. Mean sitting SBP decreased from $166.84 \pm 13.52 \mathrm{mmHg}$ at baseline to $123.05 \pm 7.83 \mathrm{mmHg}$ at the end of week 12 in group A and from $168.89 \pm 15.96 \mathrm{mmHg}$ at baseline to $130.93 \pm$ $14.84 \mathrm{mmHg}$ at the end of week 12 in group B (Table 1). Similar results were seen in SBP measurements at supine posture (Table 2). The SBP drop from baseline is superior in triple combination compared to dual combination. However both the treatments showed statistically significant reduction in SBP from baseline values ( $p<0.05$ calculated using one way ANOVA).

\subsection{The Effect of Triple Drug Combination on Diastolic Blood Pressure (DBP)}

Both the treatments showed statistically significant reduction in DBP compared to baseline values ( $\mathrm{p}<0.05$ calculated using one way ANOVA). Mean sitting DBP was decreased from $103.62 \pm 13.05 \mathrm{mmHg}$ at baseline to $81.17 \pm 8.62 \mathrm{mmHg}$ at the end of week 12 in group A. Similarly in group B, mean sitting DBP decreased from $105.43 \pm 16.51 \mathrm{mmHg}$ at baseline to $84.24 \pm 7.70 \mathrm{mmHg}$ at the end of week 12 (Table 1). Mean supine DBP also showed the similar trend which decreased from $100.22 \pm$ $10.31 \mathrm{mmHg}$ to $79.45 \pm 6.64 \mathrm{mmHg}$ in group $\mathrm{A}$ and from $103.4 \pm 10.09 \mathrm{mmHg}$ to $83.56 \pm 8.06 \mathrm{mmHg}$ in group B (Table 2).

\subsection{Effect on Quality of Life (QOL)}

The patients were asked questions based on the associated symptoms of essential hypertension, both before treatment and at the end of the treatment. The improvement was observed in symptoms such as light-headedness or faintness, sleepy feeling, weakness in limbs, blurring of vision, swelling in ankles at the end of the treatment period. Both the treatments were associated with improved QOL. 
Table 1. SBP \& DBP evaluation at sitting posture.

\begin{tabular}{ccccc}
\hline & \multicolumn{4}{c}{ Sitting } \\
\hline & \multicolumn{2}{c}{ SBP } & \multicolumn{2}{c}{ DBP } \\
\cline { 2 - 5 } & Group A & Group B & Group A & Group B \\
\hline Before Treatment (Mean \pm SD) & $166.84 \pm 13.52$ & $168.89 \pm 15.96$ & $103.62 \pm 13.05$ & $105.43 \pm 16.51$ \\
After Treatment (Mean \pm SD) & $123.05 \pm 7.83$ & $130.93 \pm 14.84$ & $81.17 \pm 8.62$ & $84.24 \pm 7.70$ \\
\hline
\end{tabular}

Table 2. SBP \& DBP evaluation at supine posture.

\begin{tabular}{ccccc}
\hline & \multicolumn{4}{c}{ Supine } \\
\hline & \multicolumn{3}{c}{ SBP } & DBP \\
\cline { 2 - 5 } & Group A & Group B & Group A & Group B \\
\hline Before Treatment (Mean \pm SD) & $165.75 \pm 15.66$ & $168.75 \pm 16.50$ & $100.22 \pm 10.31$ & $103.4 \pm 10.09$ \\
After Treatment (Mean \pm SD) & $122.5 \pm 9.38$ & $129.42 \pm 11.27$ & $79.45 \pm 6.64$ & $83.56 \pm 8.06$ \\
\hline
\end{tabular}

\subsection{Investigators' Assessment of Therapy}

Investigators' were asked to rate therapy as unsatisfactory, satisfactory, good and very good. According to investigators' rating more number of patients fell under the category of very good and good in group A compared to group B (Figure 1).

\subsection{Patients' Assessment of Therapy}

Figure 2 reveal the patients assessment of therapy based on similar ratings given above. More than $86 \%$ of patients in group A felt that the triple combination was either good or very good therapy.

\subsection{Safety Evaluations}

Both the treatments were tolerated well with few reports of adverse events. Nausea (3.77\%), vomiting (4.72\%), GI distress (3.77\%) and tiredness (3.77\%) were the major complaints from group A. Group B patients reported nausea $(5.88 \%)$, vomiting $(4.90 \%)$, headache $(4.90 \%)$ and tiredness $(6.86 \%)$ as the major problems. All the reported adverse events were of mild to moderate intensity and did not require any active management.

\section{DISCUSSION}

Intensive control of elevated blood pressure helps to prevent cardiovascular complications. Thus achieving BP goals is the major objective of the antihypertensive therapy. Monotherapy is often unsatisfactory for achieving desired BP control giving way for combinations of two or more drugs [18-20]. "Combination of the drugs having different mechanisms of action acts better in achieving BP goals" is the rationale for such combinations. The present study aimed to evaluate the effectiveness and safety of triple combination of telmisartan with amlodipine and HCT. Similar combination with olmesartan is already in the therapeutics for essential hypertension. Telmisartan is superior to olmesartan, as evident from the scientific literature thus replacing olmesartan with telmisartan in triple drug combination may have beneficial effects $[21,22]$.

The use of combination therapy in this study was consistent with the Seventh report of the Joint National Committee on prevention, detection, evaluation and treatment of high BP. It is also as per the European society of hypertension and European society of cardiology treatment guidelines. These guidelines recommend combination therapy in patients whose SBP is $>20 \mathrm{mmHg}$ above and DBP is $>10 \mathrm{mmHg}$ above the desired BP goals $[23,24]$. The availability of these drugs as a fixed dose combination in single tablet may offer additional advantage in terms of acceptance and compliance to the therapy.

The present study demonstrates the superiority of telmisartan, amlodipine and HCT triple combination over telmisartan and amlodipine combination. Both sitting and supine BP were monitored during the trial. The SBP reduced more efficiently in triple combination group than the dual combination group and the difference was statistically significant. DBP also showed similar trend in both the groups. Elevated BP hampers QOL as it is associated with many symptoms. QOL was improved in both the treatment groups as evident from the response to QOL questionnaire. The study medications were also evaluated on the basis of overall rating of therapy by investigators and also by patients. Both investigators and patients were of the opinion that triple combination of telmisartan, amlodipine and HCT is a better option than the treatment with telmisartan and amlodipine. Both the 


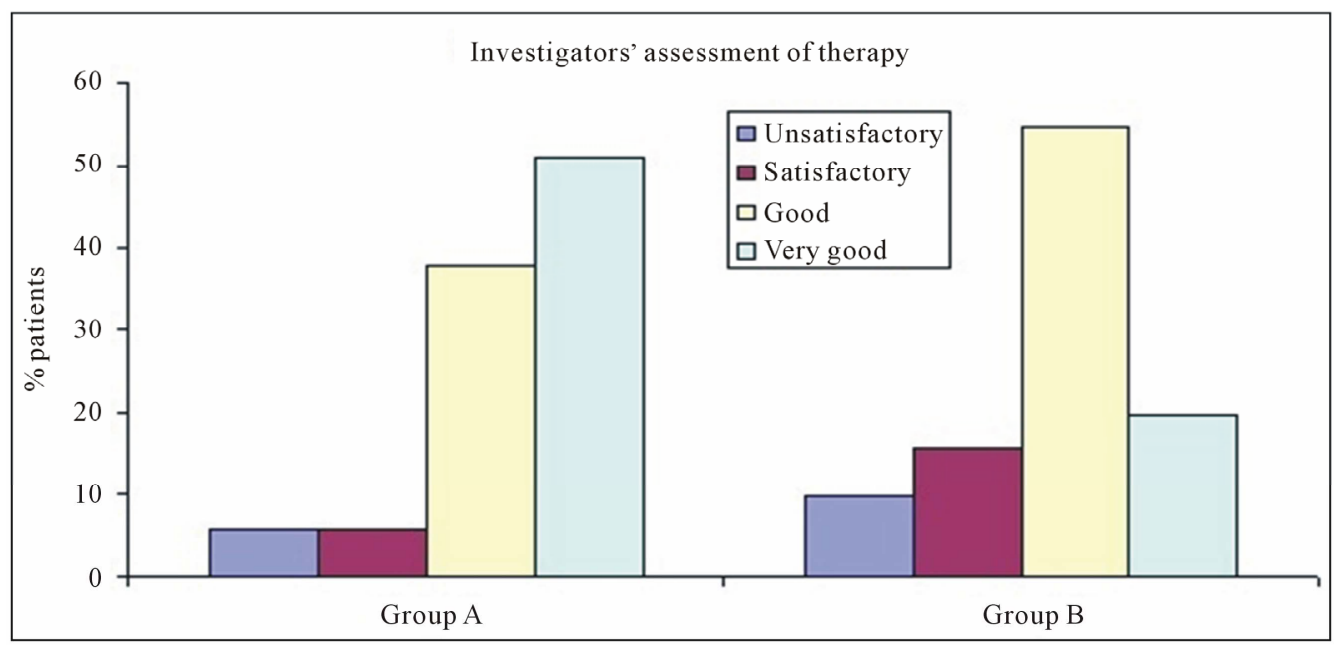

Figure 1. Investigators' assessment of therapy.

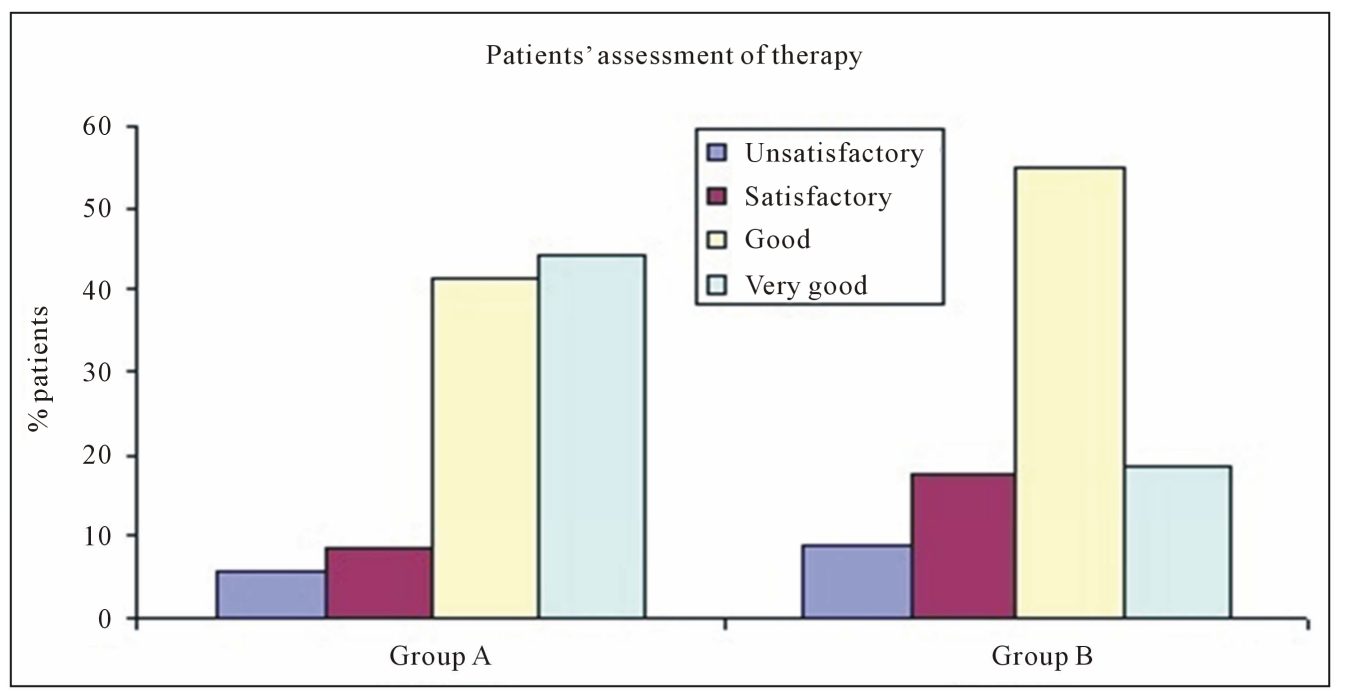

Figure 2. Patients' assessment of therapy.

treatments were tolerated well and there was no evidence of significant increase in adverse events with the addition of extra drug in triple combination.

\section{CONCLUSION}

Telmisartan when combined with amlodipine and HCT showed better control of both SBP and DBP. Thus this combination may serve a potential role in achieving desired BP goals, in patients with essential hypertension, which are otherwise poorly managed by either monotherapy or dual drug therapy. At the same time studied triple drug combination exhibits comparable tolerability profile to that of dual drug combination.

\section{REFERENCES}

[1] Pimenta, E. and Oparil, S. (2008) Fixed combinations in the management of hypertension: Patient perspectives and rationale for development and utility of the olmesartan-amlodipine combination. Vascular Health Risk Management, 4, 653-664.

[2] Swift, P.A. and MacGregor, G.A. (2002) The frequent need for three or more drugs to treat essential hypertension. What evidence for optimal combinations? Journal of Renin-Angiotensin-Aldosterone System, 3, 103-108. doi:10.3317/jraas.2002.010

[3] Plosker, G.L. and White, W.B. (2008) Telmisartan/Hydro chlorothiazide: A review of its use as fixed-dose combinations in essential hypertension. Drugs, 68, 1877-1899. doi:10.2165/00003495-200868130-00010

[4] Lacourcière, Y., Neutel, J.M. and Schumacher, H. (2005) Comparison of fixed-dose combinations of telmisartan/hydrochlorothiazide 40/12.5 mg and 80/12.5 mg and a fixed-dose combination of losartan/hydrochlorothiazide 50/12.5 mg in mild to moderate essential hypertension: pooled analysis of two multicenter, prospective, random- 
ized, open-label, blinded-end point (PROBE) trials. Clinical Therapeutics, 27, 1795-1805.

doi:10.1016/j.clinthera.2005.11.014

[5] Oparil, S., Melino, M., Lee, J., Fernandez, V. and Heyrman, R. (2010) Triple therapy with olmesartan medoxomil, amlodipine besylate, and hydrochlorothiazide in adult patients with hypertension: The TRINITY multicenter, randomized, double-blind, 12-week, parallel-group study. Clinical Therapeutics, 32, 1252-1269.

doi:10.1016/j.clinthera.2010.07.008

[6] Ram, C.V., Sachson, R., Littlejohn, T., Qian, C., Shojaee, A., Stoakes, K.A. and Neutel, J.M. (2011) Management of hypertension in patients with diabetes using an amlodipine-, olmesartan medoxomil-, and hydrochlorothiazide-based titration regimen. American Journal of Cardiology, 107, 1346-1352.

doi:10.1016/j.amjcard.2010.12.045

[7] Khan, A.H. and Imig, J.D. (2011) Telmisartan provides better renal protection than Valsartan in a rat model of metabolic syndrome. American Journal of Hypertension, 24, 816-821.

[8] Sukumaran, V., Watanabe, K., Veeraveedu, P.T., Ma, M., Gurusamy, N., Rajavel, V., Suzuki, K., Yamaguchi, K., Kodama, M. and Aizawa, Y. (2011) Telmisartan ameliorates experimental autoimmune myocarditis associated with inhibition of inflammation and oxidative stress. European Journal of Pharmacology, 652, 126-135.

[9] Maejima, Y., Okada, H., Haraguchi, G., Onai, Y., Kosuge, H., Suzuki, J.-I. and Isobe, M. (2011) Telmisartan, a unique ARB, improves left ventricular remodeling of infarcted heart by activating PPAR gamma. Laboratory Investigation, 91, 932-944. doi:10.1038/labinvest.2011.45

[10] Inoue, T., Taguchi, I., Abe, S., Toyoda, S., Sakuma, M. and Node, K. (2011) Inhibition of intestinal cholesterol absorption might explain cholesterol-lowering effect of telmisartan. Journal Clinical Pharmacy \& Therapeutics, 36, 103-110. doi:10.1111/j.1365-2710.2010.01161.x

[11] Oigman, W., Neves, M.F. and Gismondi, R.A. (2010) Combination of telmisartan plus amlodipine in the treatment of hypertension: Review of results. Expert Review of Cardiovascular Therapy, 8, 1509-1517. doi:10.1586/erc.10.143

[12] White, W.B., Littlejohn, T.W., Majul, C.R., Oigman, W., Olvera, R., Seeber, M., Schumacher, H. and Mancia, G. (2010) Effects of telmisartan and amlodipine in combination on ambulatory blood pressure in stages 1-2 hypertension. Blood Pressure Monitoring, 15, 205-212. doi:10.1097/MBP.0b013e32833c5722

[13] Lacourcière, Y. and Martin, K. (2002) Comparison of a fixed-dose combination of $40 \mathrm{mg}$ telmisartan plus 12.5 mg hydrochlorothiazide with $40 \mathrm{mg}$ telmisartan in the control of mild to moderate hypertension. American Journal of Therapeutics, 9, 111-117. doi:10.1097/00045391-200203000-00005

[14] Lacourcière, Y. (2002) A new fixed-dose combination for added blood pressure control: Telmisartan plus hydrochlorothiazide. The Journal of International Medical Research, 30, 366-379.

[15] White, W., Punzi, H., Neutel, J., Davidai, G., Koval, S. and Murwin, D. (2005) Telmisartan plus hydrochlorothiazide (80/25 mg) has a greater antihypertensive effect than valsartan plus hydrochlorothiazide (160/25 mg) in patients with stage 1 and 2 hypertension. American Journal of Hypertension, 18, 97A. doi:10.1016/j.amjhyper.2005.03.271

[16] Mason, R.P. (2002) Mechanisms of plaque stabilization for the dihydropyridine calcium channel blocker amlodipine: Review of the evidence. Atherosclerosis, 165, 191. doi:10.1016/S0021-9150(01)00729-8

[17] De Portu, S. and Mantovani, L.G. (2009) Amlodipine: A pharmacoeconomic review. Journal of Medical Economics, 12, 60-68. doi:10.3111/13696990802525266

[18] Nakazato, T., Kawada, T., Shigematsu, T. and Yamada, K. (2002) Left ventricular hypertrophy was infrequent in patients starting dialysis after undergoing a strict blood pressure control in the pre-dialytic period. Internal Medicine, 41, 925-930. doi:10.2169/internalmedicine.41.925

[19] Rakugi, H., Ogihara, T., Goto, Y. and Ishii, M. (2010) Comparison of strict- and mild-blood pressure control in elderly hypertensive patients: A per-protocol analysis of JATOS. Hypertension Research, 33, 1124-1128. doi:10.1038/hr.2010.144

[20] Sato, A., Watanabe, S., Okubo, S., Toi, T., Nakano, H., Tada, J. and Aonuma, K. (2010) The therapeutic importance of home blood pressure assessment and combination antihypertensive therapy for achieving target blood pressure control: Ibaraki hypertension assessment trial. Hypertension Research, 33, 1264-1271.

doi:10.1038/hr.2010.175

[21] Galzerano, D., Capogrosso, C., Michele, S.D., Galzerano, A., Paparello, P., Lama, D. and Gaudio, C. (2010) New standards in hypertension and cardiovascular risk management: Focus on Telmisartan. Vascular Health and Risk Management, 6, 113-133. doi:10.2147/VHRM.S7857

[22] Fogari, R., Zoppi, A., Mugellini, A., Preti, P., Destro, M., Rinaldi, A. and Derosa, G. (2008) Effectiveness of hydrochlorothiazide in combination with telmisartan and olmesartan in adults with moderate hypertension not controlled with monotherapy: A prospective, randomized, open-label, blinded end point (PROBE), parallel-arm study. Current Therapeutic Research, 69, 1-15. doi:10.1016/j.curtheres.2008.02.003

[23] Chobanian, A.V., Bakris, G.L., Black, H.R., Cushman, W.C., Green, L.A., Izzo, J.L. Jr., Jones, D.W., et al. (2003) The seventh report of the joint national committee on prevention, detection, evaluation, and treatment of high blood pressure: The JNC 7 report. Journal of American Medical Association, 21, 2560-2572. doi:10.1001/jama.289.19.2560

[24] Mancia, G., De Backer, G., Dominiczak, A., Cifkova, R., Fagard, R., Germano, G., Grassi, G., et al. (2007) Guidelines for the management of arterial hypertension: The task force for the management of arterial hypertension of the European Society of Hypertension (ESH) and of the European Society of Cardiology (ESC). European Heart Journal, 28, 1462-1536. 